\title{
Autoimmune Hemolytic Anemia in a 24-Year-Old Patient With COVID-19 Complicated by Secondary Cryptococcemia and Acute Necrotizing Encephalitis: A Case Report and Review of Literature
}

\author{
Indryas L. Woldie ${ }^{\mathrm{a}, \mathrm{d}}$, Ian G. Brown ${ }^{\mathrm{b}, \mathrm{d}}$, Nduka Francis Nwadiaro ${ }^{\mathrm{b}}$, Amit Patel ${ }^{\mathrm{b}}$, \\ Mohammad Jarrar $^{\mathrm{a}}$, Elise Quint ${ }^{\mathrm{c}}$, Vladislav Khokhotva ${ }^{\mathrm{b}}$, Nicole Hugel ${ }^{\mathrm{b}}$, \\ Michael Winger ${ }^{\mathrm{b}}$, Alexander Briskin ${ }^{\mathrm{b}}$
}

\begin{abstract}
Since the initial reported outbreak of coronavirus disease 2019 (COVID-19), many unique case reports have been published in the medical literature. Here we report a complicated clinical course of a young patient with COVID-19 who presented initially with recurrent autoimmune hemolytic anemia (AIHA). He subsequently developed bilateral pulmonary emboli, and ultimately succumbed to encephalitis and cryptococcemia in the context of being treated with high dose immunosuppression for the AIHA. Combining immunosuppression with active COVID-19 infection presents some truly challenging diagnostic and management scenarios which this case summarizes and highlights very well. Based on this case, we propose some strategies on how to approach these difficult decisions while also recognizing the significant gaps that exist in such an evolving topic. Lastly, this case also represents a potentially novel presentation of secondary fungal infection of the central nervous system (CNS) related to COVID-19.
\end{abstract}

Keywords: COVID-19; Autoimmune hemolytic anemia; Acute necrotizing encephalitis; Cryptococcemia

\section{Introduction}

Since the initial reported outbreak of coronavirus disease 2019 (COVID-19), many different complications of the virus have

Manuscript submitted August 28, 2020, accepted September 10, 2020

Published online September 23, 2020

\footnotetext{
${ }^{a}$ Windsor Regional Cancer Center, Windsor, ON, Canada

${ }^{b}$ Windsor Regional Hospital, Windsor, ON, Canada

${ }^{\text {'S }}$ chulich School of Medicine, Western University, London, ON, Canada

${ }^{\mathrm{d} C o r r e s p o n d i n g ~ A u t h o r s: ~ I a n ~ G . ~ B r o w n ~ a n d ~ I n d r y a s ~ L . ~ W o l d i e, ~ W i n d s o r ~ R e-~}$ gional Hospital/Cancer Center, 1995 Lens Ave., Windsor, ON N8W 1L9, Canada. Emails: ian.brown@wrh.on.ca and indriaslw@yahoo.com
}

doi: https://doi.org/10.14740/jmc3575 been identified including but not limited to worsening of underlying comorbidities [1], venous thromboembolism (VTE) [2], and secondary infection [3]. This case highlights all of these complications with some unique aspects which make diagnosis and management especially challenging.

COVID-19 has been shown to be a trigger for exacerbating auto-immune cytopenias [1] and it's still not entirely clear how to approach immunosuppression and what additional risk factors may be present in patients with COVID-19. This case walks through that decision-making process and retrospectively serves as a reminder of the risks of high dose immunosuppression.

It has also been well documented that patients with COVID-19 are at risk for VTE [2], but how long that risk persists, and how to stratify these patients in the outpatient setting is still undefined and needs further study.

COVID-19 infections can present in a wide variety of ways and can affect virtually any organ system, including the central nervous system (CNS). Viral encephalitis related to COVID-19 has been described but is not a well-defined entity at this point. Furthermore, acutely ill patients with COVID-19 are known to be at high risk for secondary infections, so having a broad differential is essential. This unique case highlights the approach to CNS presentations in patients with COVID-19 and examines what role COVID-19 might play with secondary cryptococcemia and meningoencephalitis.

\section{Case Report}

\section{Investigations}

A 24-year-old male presented to a rural hospital with a 2-day history of subjective fevers, myalgias, and dry cough. His past medical history was significant for autoimmune hemolytic anemia (AIHA) which had been diagnosed 3 years prior. In the years since that diagnosis, he had remained stable on chronic prednisone (20 mg daily) with no flares of anemia. He had no relevant family history, and he worked in agriculture. On ex- 
amination, he was febrile at $38.5^{\circ} \mathrm{C}$ but exam was otherwise normal including an oxygen saturation of $98 \%$ on room air.

\section{Diagnosis}

Laboratory tests showed a hemoglobin of $75 \mathrm{~g} / \mathrm{L}$ (reference range (RR): 135 - $165 \mathrm{~g} / \mathrm{L}$ ), an elevated unconjugated bilirubin of $41 \mu \mathrm{mol} / \mathrm{L}$ (RR: 0 - $20 \mu \mathrm{mol} / \mathrm{L}$ ), and elevated lactate dehydrogenase of $347 \mathrm{U} / \mathrm{L}(\mathrm{RR}:<234 \mathrm{U} / \mathrm{L})$. A direct antiglobulin test (DAT) came back positive (immunoglobulin G (IgG) and complement coating red cells). A nasopharyngeal swab was positive for COVID-19 (Roche LC480 real-time polymerase chain reaction (PCR)). A diagnosis of AIHA and active COVID-19 infection was made.

\section{Treatment}

The patient was placed on prednisone $(1.5 \mathrm{mg} / \mathrm{kg})$ and admitted to hospital. His hemoglobin continued to drop to as low as $58 \mathrm{~g} / \mathrm{L}$ over the next $24 \mathrm{~h}$ and he was transferred to our tertiary center. He was now afebrile and completely asymptomatic. Steroids were continued, and cyclophosphamide was added (50 mg per os (PO) bis in die (BID)). Subsequently, his hemoglobin and reticulocyte response started to gradually go up. The patient was discharged on day 13 of admission with a hemoglobin of $85 \mathrm{~g} / \mathrm{L}$ with a plan to taper his prednisone as an out-patient.

\section{Follow-up and outcomes}

One week later at his follow-up appointment, the patient complained of a persistent headache. There were no focal neurological symptoms or deficits, and the only abnormal physical exam finding was a heart rate of 122 . His hemoglobin was $114 \mathrm{~g} / \mathrm{L}$ and a repeat nasopharyngeal swab for COVID-19 was negative. An urgent computed tomography (CT) head and CT chest (pulmonary embolism protocol) were ordered. The CT chest showed bilateral subsegmental pulmonary emboli as well as faint bilateral ground glass opacities. The CT head showed non-specific symmetric cerebral enhancement. The patient was readmitted and started on full dose anticoagulation (dalteparin $200 \mathrm{U} / \mathrm{kg}$ daily). A repeat COVID-19 nasopharyngeal swab (now 27 days after his initial symptoms) was positive once again.

Shortly after readmission, the patient developed decreased level of consciousness and seizure activity. A statim (STAT) magnetic resonance imaging (MRI) of the brain showed T2/fluid-attenuated inversion recovery (FLAIR) hyper intensity on the medial aspect of each temporal lobe, bilateral basal ganglia and medial thalami consistent with severe acute necrotizing encephalitis (Fig. 1). Of note, COVID-19 encephalitis was specifically mentioned in the MRI report as a diagnostic possibility. Magnetic resonance angiography and venography of the brain showed normal cerebral vasculature. A working diagnosis of COVID-19 encephalitis was established, and an urgent lumbar puncture was planned, however the patient sustained a cardiac arrest with

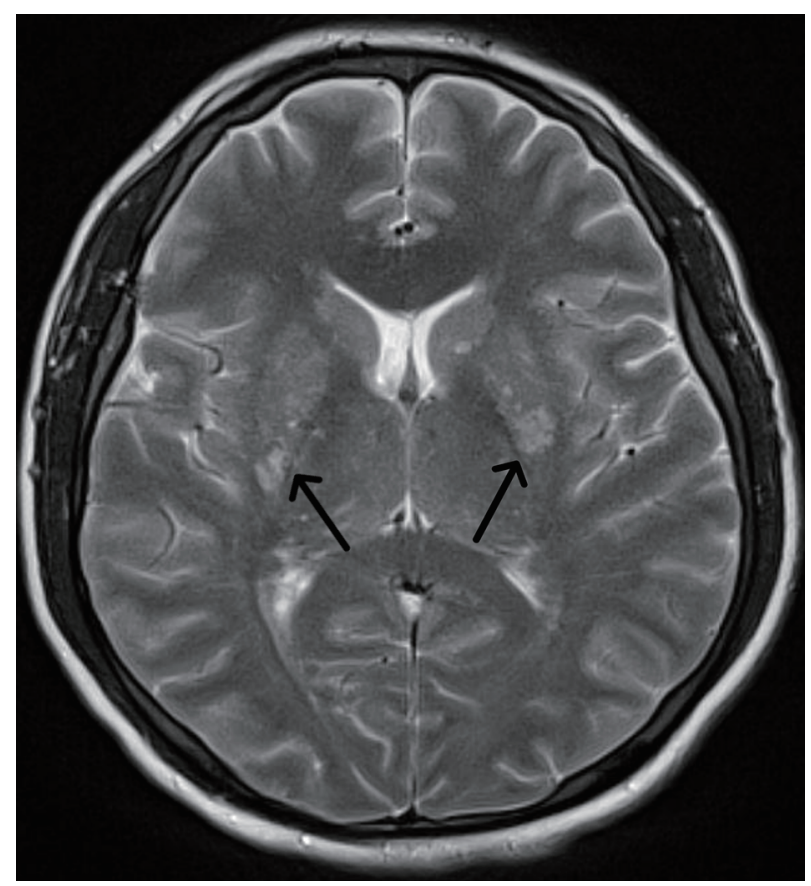

Figure 1. T2-weighted MRI showing abnormal signal in the basal ganglia (arrows). MRI: magnetic resonance imaging.

pulseless electrical activity (PEA) prior to the procedure. Return of spontaneous circulation was achieved but the patient was now intubated and requiring vasopressor support. Methylprednisolone (1 g intravenous (IV) daily) and broad-spectrum antibiotics were initiated along with IVIG $(1 \mathrm{~g} / \mathrm{kg})$. Sadly, the patient's mental status did not improve, and life support measures were later withdrawn after discussions with the family.

Three days later, two blood cultures came back positive for Cryptococcus neoformans (when earlier blood cultures during the admission had been negative). Acute-on-chronic immunosuppression from steroids, along with his occupational exposure (farming), had likely precipitated a fulminant Cryptococcus infection. It was unclear if his encephalitis was completely or partially related to cryptococcal meningoencephalitis, or how significantly COVID-19 may have been involved.

\section{Discussion}

This case provides a very broad overview of COVID-19-related complications and demonstrates how complex the clinical decision making can become. These decisions are made more difficult by the fact that there is fairly limited evidence that currently exists in the literature. The case allows us to explore some of the relevant literature and attempt to apply it to the clinical scenarios that we were faced with. We also discuss the thought process at various points in the patient's course to hopefully provide a general approach when clinicians are struggling with similar COVID-19-related decisions.

Warm and cold AIHA, immune thrombocytopenia, and Evan's syndrome have all been described in association with COVID-19 infection $[1,4,5]$. In a case series of seven patients 
with warm and cold AIHA and COVID-19 infection, steroids were used in five of the patients, with Rituximab in two patients who had inadequate response to steroids [1]. In general, the efficacy of steroids (or rituximab) for immune-mediated cytopenias is clear, but what remains unclear is the impact of these immunosuppressants on patients with COVID-19. In our case, we considered Rituximab, but given the relative stability of the patient's hemoglobin, we decided against it due to the uncertainty surrounding safety data in this setting. Splenectomy was also considered, but surgical risks were deemed unacceptably high. Specific risks included risk of VTE, potential healthcare exposures to COVID-19, and transfusion limitations in the setting of autoimmune anemia and blood matching with an already anemic patient. With our patient being young and otherwise healthy, and having only modest symptoms of COVID-19, we elected to start steroids (and later the cyclophosphamide). At the time of this patient's admission, it was largely unknown what effect steroids might have on the progression of COVID-19. Since then, evidence has surfaced that steroids may in fact be beneficial to patients with severe COVID-19 manifestations. Our patient did not have severe symptoms, and the potential benefit of steroid use in this population is not clear. Thus, we can only state that decisions regarding immunosuppression in the setting of active COVID-19 infection take into account the risks and benefits as per usual.

The prevalence of VTE in patients with severe COVID-19 infection has been shown to be anywhere between $25-42 \%$ in various studies [6,7]. This has resulted in various different recommendations by several institutions regarding prophylactic or therapeutic anticoagulation in patients with COVID-19 but there is no clear consensus. Furthermore, there are no recommendations around VTE prophylaxis postdischarge in these patients. Our patient was on VTE prophylaxis (dalteparin 5,000 U omne in die (OD)) during his initial hospitalization, and even had negative Doppler ultrasounds of both legs (ordered because his D-dimer was elevated). He subsequently presented to hospital a week later with bilateral pulmonary emboli. His sinus tachycardia was the only manifestation and serves as a reminder to have a high index of suspicion for VTE in these patients. Additionally, given the aforementioned high probability for deep vein thrombosis (DVT) or pulmonary embolism (PE) in these patients, we would propose that the threshold for ordering investigations should be lower than the general population. There is no evidence to suggest that our patient should have been on VTE prophylaxis after his initial discharge, but potentially this is an area for further study given the high risk of VTE in patients with COVID-19.

Neurological manifestations are reported to occur in up to $25 \%$ of patients with COVID-19 [8]. These include anosmia, dysgeusia, febrile seizures, convulsions, change in mental status and encephalitis. Etiologies behind these various neurological complications have also been described including viral encephalitis (with COVID-19 identified in the cerebrospinal fluid (CSF)), toxic encephalopathy, and cerebrovascular disease. In the few reported cases of COVID-19 encephalitis, intracranial cytokine storm has been proposed as a mechanism, and IVIG along with high dose steroids have been tried as treatment although efficacy is unknown [8]. The additional finding of Cryptococcus neoformans in the blood culture of our patient raises a strong suspicion for cryptococcal meningoencephalitis. Unfortunately, this was not definitively confirmed without CSF analysis or autopsy. The high dose steroids for his recurrent AIHA (on top of his baseline chronic immunosuppression) were likely the main precipitant for the invasive fungal infection. It is unclear if active COVID-19 infection and/ or COVID-19 encephalitis may have also been contributing, or if the presence of COVID-19 infection further predisposed this patient to opportunistic infection. The MRI findings seen in our patient were consistent with previous case reports of necrotizing encephalitis from COVID-19. These typical findings have been described as symmetric multifocal lesions with invariable thalamic involvement [9]. As was mentioned in the case, the radiology report specifically singled out COVID-19 necrotizing encephalitis as a diagnostic possibility. However, we now make a point of stating that these MRI findings are not specific to COVID-19 and can be seen in a variety of conditions including virtually any CNS infection. In hindsight, the positive diagnosis of COVID-19 along with the MRI report may have led to a case of tunnel vision and anchoring. Our patient's neurological decline was attributed to COVID-19 encephalitis being exacerbated by immunosuppression, and opportunistic infections were not immediately considered. The unfortunate reminder is to always be aware of secondary infection particularly in the context of immunosuppression, regardless of COVID-19 status. In terms of risk of developing secondary infections, a recent case series from Wuhan, China showed that $25.5 \%$ and $10.9 \%$ of critically ill patients hospitalized with COVID-19 had bacterial and fungal coinfection, respectively [10]. Thus, the risk is quite high, and it is something that many clinicians may not be aware of. To our knowledge, no secondary CNS infections or cryptococcemia have been described with COVID-19 to date. Ultimately, we are unable to conclude if our patient's encephalitis was entirely due to Cryptococcus, COVID-19, or a combination of both.

The major limitation of this report is the lack of definitive diagnosis, particularly for the CNS manifestations in our patient. Having CSF analysis and/or postmortem examination would have been helpful to make conclusions about any potential relationship between COVID-19 and cryptococcal meningoencephalitis in this patient. In addition, the lack of previous experience and guidelines in managing complications related to COVID-19 is a major constraint and makes it very difficult to offer any firm recommendations. In general, we are left to fall back on the fundamentals of diagnosis and management. Namely, always consider the implications of treatment (and be prepared to act on those potential complications), and always aim to make patient centered clinical decisions.

\section{Learning points}

COVID-19 infections can predispose patients to many complications including but not limited to worsening of underlying medical conditions, VTE, and secondary infections. Clinicians must be mindful of these complications during each patient encounter (both in the inpatient and outpatient settings). The potential benefit of immunosuppression in the context of COVID-19 (or any active infection) needs to strongly be weighed 
against the risks. Neurologic symptoms and presentations related to COVID-19 are not uncommon, but clinicians must keep a broad differential and avoid anchoring bias. Lastly, opportunistic infections should always be on the differential of the acutely ill patient in the context of potent immunosuppression.

\section{Acknowledgments}

None to declare.

\section{Financial Disclosure}

None to declare.

\section{Conflict of Interest}

None to declare.

\section{Informed Consent}

The manuscript has been de-identified, and the consent could not be obtained as the patient passed away, and no family were reachable at the time of writing the manuscript.

\section{Author Contributions}

All authors were directly involved in patient care and contributed to writing and editing the manuscript.

\section{Data Availability}

The authors declare that data supporting the findings of this study are available within the article.

\section{References}

1. Lazarian G, Quinquenel A, Bellal M, Siavellis J, Jacquy C, Re D, Merabet F, et al. Autoimmune haemolytic anaemia associated with COVID-19 infection. Br J Haematol. 2020;190(1):29-31.

2. Cui S, Chen S, Li X, Liu S, Wang F. Prevalence of venous thromboembolism in patients with severe novel coronavirus pneumonia. J Thromb Haemost. 2020;18(6):14211424.

3. Zhou F, Yu T, Du R, Fan G, Liu Y, Liu Z, Xiang J, et al. Clinical course and risk factors for mortality of adult inpatients with COVID-19 in Wuhan, China: a retrospective cohort study. Lancet. 2020;395(10229):1054-1062.

4. Li M, Nguyen CB, Yeung Z, Sanchez K, Rosen D, Bushan S. Evans syndrome in a patient with COVID-19. Br J Haematol. 2020;190(2):e59-e61.

5. Zulfiqar AA, Lorenzo-Villalba N, Hassler P, Andres E. Immune thrombocytopenic purpura in a patient with COVID-19. N Engl J Med. 2020;382(18):e43.

6. Klok FA, Kruip M, van der Meer NJM, Arbous MS, Gommers D, Kant KM, Kaptein FHJ, et al. Incidence of thrombotic complications in critically ill ICU patients with COVID-19. Thromb Res. 2020;191:145-147.

7. Wu Y, Xu X, Chen Z, Duan J, Hashimoto K, Yang L, Liu $\mathrm{C}$, et al. Nervous system involvement after infection with COVID-19 and other coronaviruses. Brain Behav Immun. 2020;87:18-22.

8. Poyiadji N, Shahin G, Noujaim D, Stone M, Patel S, Griffith B. COVID-19-associated acute hemorrhagic necrotizing encephalopathy: imaging features. Radiology. 2020;296(2):E119-E120.

9. Zhang G, Hu C, Luo L, Fang F, Chen Y, Li J, Peng Z, et al. Clinical features and short-term outcomes of 221 patients with COVID-19 in Wuhan, China. J Clin Virol. 2020;127:104364. 